\title{
Successful treatment for psychomotor agitation in neuromyelitis optica spectrum disorder with trazodone-risperidone combination: a case report
}

This article was published in the following Dove Press journal:

Neuropsychiatric Disease and Treatment

13 March 2017

Number of times this article has been viewed

\author{
Zui Narita' \\ Harumasa Takano' \\ Tomiki Sumiyoshi \\ 'Department of Psychiatry, National \\ Center Hospital, ${ }^{2}$ Department of \\ Clinical Epidemiology, Translational \\ Medical Center, National Center \\ of Neurology and Psychiatry, \\ Tokyo, Japan
}

\begin{abstract}
Neuromyelitis optica (NMO) is a relapsing disease that typically affects the spinal cord and optic nerves. So far, a few studies have reported pharmacologic treatment for psychiatric symptoms in patients with NMO spectrum disorder (NMOSD). However, no literature has described psychomotor agitation associated with the disease and its treatment. We report an 84-year-old woman with NMOSD whose psychomotor agitation was effectively treated with a combination of trazodone and risperidone. Our observation suggests the ability of augmentation of antipsychotic drugs with antidepressants to ameliorate psychotic symptoms associated with NMOSD.
\end{abstract}

Keywords: neuromyelitis optica, multiple sclerosis, psychomotor agitation, trazodone, risperidone

\section{Introduction}

Neuromyelitis optica (NMO) is an autoimmune condition of the central nervous system, which is characterized by optic neuritis and longitudinal transverse myelitis. ${ }^{1,2}$ Its diagnosis is based on clinical symptoms and the presence of serum antiaquaporin-4 antibody. ${ }^{3}$ The incidence of NMO per 100,000 population ranges from 0.05 to 0.4 , while the prevalence per 100,000 population ranges from 0.052 to $4.4 .^{4}$ Multiple sclerosis (MS) is sometimes misdiagnosed in patients with NMO. ${ }^{5}$ With systematic literature reviews and electronic surveys to facilitate consensus, the International Panel for NMO Diagnosis (IPND) was convened to develop revised diagnostic criteria, which defines the unifying concept, NMO spectrum disorder (NMOSD) ${ }^{6}$

So far, a few studies have reported psychiatric symptoms in patients with NMO or NMOSD. ${ }^{1,7,8}$ For example, Woolley et $\mathrm{al}^{1}$ found that depressive symptoms and primary polydipsia were effectively treated with sertraline and aripiprazole in the case of NMO. Another study suggested that moderate to severe depression in patients with NMOSD is associated with neuropathic pain and fatigue, which tend to be insufficiently treated. ${ }^{8}$ On the other hand, Alam et $\mathrm{al}^{7}$ observed that psychotic symptoms and catatonia because of the illness were ameliorated with risperidone and lorazepam. However, there has been no report of psychomotor agitation associated with NMOSD or its successful treatment.

Here, we report a patient with NMOSD whose psychomotor agitation was effectively treated with the combination of trazodone and risperidone. The patient has provided a written informed consent for the case details and the accompanying images to be published.
Department of Psychiatry, National

Center of Neurology and Psychiatry

4-I-I, Ogawahigashi, Kodaira,

Tokyo I87-855I, Japan

$\mathrm{Tel}+8|4234| 27||$

Fax +81423461944

Email zuinarita@ncnp.go.jp 


\section{Case}

Mrs A was an 84-year-old Japanese woman. One year before consulting our hospital, she realized that her visual acuity was impaired. When she was admitted to the previous hospital, she showed blindness with light perception. Serum antiaquaporin-4 antibody was positive by enzyme-linked immunosorbent assay. In addition, brain magnetic resonance imaging (MRI) showed right optic neuritis and did not meet Paty's diagnostic criteria for MS, indicating the diagnosis of NMOSD based on 2015 IPND criteria. She had been treated with $8 \mathrm{mg} / \mathrm{d}$ prednisolone and had been mentally stable for about 1 year. However, she suddenly began to cry loudly and became aggressive to her husband. Moreover, she became violent and began slapping her husband occasionally. Olanzapine was not effective in treating these symptoms, which encouraged her to consult us.

She showed bilateral total blindness without light perception when admitted to our hospital. Brain MRI revealed right optic neuritis (Figure 1), although it was unclear whether it was a new one or not (gadolinium enhancing scan was not performed). Psychomotor agitation was noted; she was irritable, yelling "Do it quicker!" and hitting nurses repeatedly.

As "other mental disorders due to NMOSD" were diagnosed in Mrs A, we started treating her with prednisolone, methotrexate, and risperidone. Risperidone, at $2 \mathrm{mg} / \mathrm{d}$, was

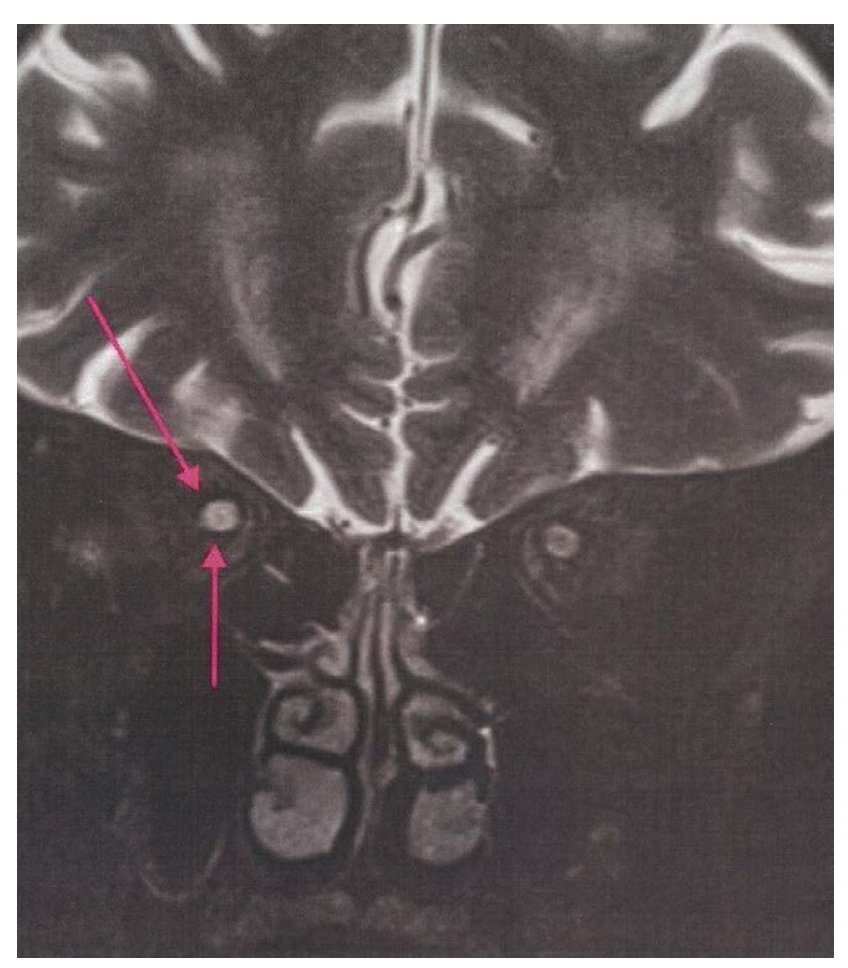

Figure I Brain magnetic resonance imaging of the patient.

Note: The coronal view of T2-weighted image. The arrows indicate high signal intensity in the right optic nerve. only partially effective, but she could not tolerate the dose of $3 \mathrm{mg} / \mathrm{d}$ because of occurrence of dysphagia. Therefore, we added trazodone at $25 \mathrm{mg} / \mathrm{d}$ instead of titrating risperidone. After maintaining the combination dose of trazodone and risperidone for 3 days, she no longer shouted out loud or resorted to violence. At the time of discharge, she was more observant and insightful enough to be adherent to medications.

\section{Discussion}

To our knowledge, this is the first report of a case of NMOSD eliciting psychomotor agitation.

Several psychiatric conditions deserve considerations for differential diagnosis. These may include delirium, which is unlikely given that Mrs A had been fully conscious throughout the time course and that electroencephalography recordings were normal. Substance- or medication-induced agitation is not probable either in view of the absence of a recent history of substance use or change in medications. In addition, since steroid-induced psychiatric symptoms typically develop over the dose of $40 \mathrm{mg} / \mathrm{d}$, administering a small dose $(8 \mathrm{mg} / \mathrm{d})$ of prednisolone was unlikely to cause psychomotor agitation. $^{9}$

Behavioral and psychological symptoms of dementia can be excluded, as her cognitive function was not impaired. This was supported by perfect performance on the Mini-Mental State Examination except in subtests requiring visual acuity, ie, those that are not affected by blindness. Taken together, the psychotic symptoms of the patient were likely to be related to neuroimmunological malfunctions associated with NMOSD.

We also provide the first report of the effectiveness of trazodone on psychiatric symptoms due to NMOSD. This antidepressant is often used to treat delirium because of sleepinducing effects and a small likelihood of causing adverse events. ${ }^{10}$ The hypnotic effect of trazodone is induced by its property as a serotonin reuptake inhibitor and antagonist at serotonin/adrenaline/histamine receptors. ${ }^{11,12}$ The use of antipsychotics is generally associated with side effects, such as extrapyramidal symptoms, especially at a high dose. ${ }^{13}$ Hence, the successful treatment with the combination of trazodone and low-dose risperidone, reported here, may provide a strategy for ameliorating psychomotor agitation in NMOSD.

\section{Conclusion}

This case report indicates the efficacy of the combination of trazodone and an antipsychotic drug on psychomotor agitation associated with NMOSD. 


\section{Disclosure}

The authors report no conflicts of interest in this work.

\section{References}

1. Woolley J, Douglas VC, Cree BA. Neuromyelitis optica, psychiatric symptoms and primary polydipsia: a case report. Gen Hosp Psychiatry. 2010;32(6):648.e5-648.e8.

2. Jarius S, Wildemann B, Paul F. Neuromyelitis optica: clinical features, immunopathogenesis and treatment. Clin Exp Immunol. 2014; 176(2):149-164.

3. Zekeridou A, Lennon VA. Aquaporin-4 autoimmunity. Neurol Neuroimmunol Neuroinflamm. 2015;2(4):e110.

4. Pandit L, Asgari N, Apiwattanakul M, et al. Demographic and clinical features of neuromyelitis optica: a review. Mult Scler. 2015;21(7): $845-853$.

5. Metz I, Beißbarth T, Ellenberger D, et al. Serum peptide reactivities may distinguish neuromyelitis optica subgroups and multiple sclerosis. Neurol Neuroimmunol Neuroinflamm. 2016;3(2):e204.

6. Wingerchuk DM, Banwell B, Bennett JL, et al. International consensus diagnostic criteria for neuromyelitis optica spectrum disorders. Neurology. 2015;85(2):177-189.
7. Alam A, Patel R, Locicero B, Rivera N. Neuromyelitis optica presenting with psychiatric symptoms and catatonia: a case report. Gen Hosp Psychiatry. 2015;37(3):274.e1-274.e2.

8. Chavarro VS, Mealy MA, Simpson A, et al. Insufficient treatment of severe depression in neuromyelitis optica spectrum disorder. Neurol Neuroimmunol Neuroinflamm. 2016;3(6):e286.

9. Kenna HA, Poon AW, de los Angeles CP, Koran LM. Psychiatric complications of treatment with corticosteroids: review with case report. Psychiatry Clin Neurosci. 2011;65(6):549-560.

10. Okamoto Y, Matsuoka Y, Sasaki T, et al. Trazodone in the treatment of delirium. J Clin Psychopharmacol. 1999;19(3):280-282.

11. Morin AK, Jarvis CI, Lynch AM. Therapeutic options for sleepmaintenance and sleep-onset insomnia. Pharmacotherapy. 2007; 27(1):89-110.

12. Benca RM. Diagnosis and treatment of chronic insomnia: a review. Psychiatr Serv. 2005;56(3):332-343.

13. Saltz BL, Woerner MG, Robinson DG, Kane JM. Side effects of antipsychotic drugs. Avoiding and minimizing their impact in elderly patients. Postgrad Med. 2000;107(2):169-172.
Neuropsychiatric Disease and Treatment

\section{Publish your work in this journal}

Neuropsychiatric Disease and Treatment is an international, peerreviewed journal of clinical therapeutics and pharmacology focusing on concise rapid reporting of clinical or pre-clinical studies on a range of neuropsychiatric and neurological disorders. This journal is indexed on PubMed Central, the 'PsycINFO' database and CAS,

\section{Dovepress}

and is the official journal of The International Neuropsychiatric Association (INA). The manuscript management system is completely online and includes a very quick and fair peer-review system, which is all easy to use. Visit http://www.dovepress.com/testimonials.php to read real quotes from published authors. 